\title{
Protée
}

\section{La rumeur}

\section{Parole fragile et croyance partagée}

\section{Josias Semujanga}

Volume 32, numéro 3, hiver 2004

La rumeur

URI : https://id.erudit.org/iderudit/011257ar

DOI : https://doi.org/10.7202/011257ar

Aller au sommaire du numéro

\section{Éditeur(s)}

Département des arts et lettres - Université du Québec à Chicoutimi

ISSN

0300-3523 (imprimé)

1708-2307 (numérique)

Découvrir la revue

Citer cet article

Semujanga, J. (2004). La rumeur : parole fragile et croyance partagée. Protée, 32(3), 33-46. https://doi.org/10.7202/011257ar

\section{Résumé de l'article}

Cette étude se fait en trois moments. En premier lieu, elle montre les limites des approches béhavioristes et sociologiques abordant la rumeur sous l'angle clinique comme si elle était une pathologie dont il fallait débarrasser la société. Ensuite, elle propose une analyse centrée sur la théorie de l'énonciation à partir des hypothèses avancées par la sémiotique du discours (Bertrand), qui stipulent que toute énonciative individuelle se construit à partir de l'énonciation collective envisagée comme schéma où se trouvent déposés sous formes de sédiments certains énoncés figés comme des stéréotypes et des clichés. Enfin, il en découle une instruction que toute parole a une visée argumentative dont le procès se construit à partir des valeurs partagées entre les interlocuteurs. Car, en convoquant les clichés et les stéréotypes du thésaurus social, la narration d'une rumeur publique vise l'adhésion des interlocuteurs sur la base d'un schéma axiologique binaire marquant, de manière simple et claire, la frontière entre le bien et le mal, l'acceptable et le condamnable. L'analyse illustrera ce cadre théorique par l'étude d'une rumeur spécifique : le " complot tutsi » dans le discours sur le Rwanda avant et après le génocide de 1994.
Ce document est protégé par la loi sur le droit d'auteur. L'utilisation des services d’Érudit (y compris la reproduction) est assujettie à sa politique d'utilisation que vous pouvez consulter en ligne.

https://apropos.erudit.org/fr/usagers/politique-dutilisation/ 


\section{LA RU M EU R \\ PARO LE FRAGILE ET CRO YAN CE PARTAGÉE}

Jo SIAS SEM UJANGA

La rumeur fait surgir, en une étroite relation, une série d'évocations à la mesure de son sens indéterminé et qui change selon la situation de communication qui la prend en charge. Car si le terme est habituellement employé pour décrire la circulation (souvent rapide, comme une épidémie, on parle parfois de communication virale) d'informations incontrôlées, il désigne aussi une information qui reste à prouver, dont on ne connait pas la source ${ }^{1}$. Son usage signalerait, dans le discours social où s'expriment les rapports humains, une société en crise de valeurs où les gens auraient la propension à raconter des histoires non vérifiables. La rumeur s'entend effectivement comme une nouvelle d'origine mystérieuse ou dissimulée, qui se répand amplement sans être examinée. Aussi, aucune société ne peut-elle, à elle seule, en réclamer l'invention, puisque la rumeur a partie liée avec l'usage des récits dans toute communauté humaine. Son usage revêt une dimension transculturelle. Parole incontrôlable, la rumeur se construit par rapport à d'autres formes stéréotypées et figées reconnaissables dans le discours social. Elle procède donc des mécanismes de l'énonciation et fait partie des stratégies de l'argumentation, usant abondamment de préjugés et de clichés tombés au statut de résidus discursifs dans le discours social.

À ce statut discursif de la rumeur s'ajoute celui de sa forme, puisque le mot rumeur a longtemps été utilisé pour désigner un récit de type oral, excluant du même coup toute possibilité pour l'image et l'écrit de médiatiser la rumeur. On admet cependant, depuis un certain temps, que la rumeur puisse s'appliquer à d'autres domaines comme l'écrit, l'image, la caricature, surtout avec l'avènement d'Internet (Froissart, 2002). Mais le plus souvent on ne remet pas en question l'unicité de la forme du récit, qui est fondée sur une narration utilisant habituellement le canal informel du bouche à oreille (médium temporaire, qui s'oppose en particulier à la permanence de l'écrit ou de l'image).

Nous allons d'abord analyser les modalités énonciatives de la véridiction rumorale, en rappelant brièvement les rapports entre l'argumentation et l'énonciation, deux fondements de la narration de la rumeur, puis nous allons analyser une rumeur spécifique, le "complot tutsi», dans le discours sur le Rwanda avant et après le génocide de 1994. 


\section{LA RUMEUR DANS LES ENJEUX DU DISCOURS} OU LE MIROITEMENT DE LA VÉRIDICTION

Comme elle semble procéder de l'idée de fiction/ vérité, toute analyse de la rumeur relève d'une typologie générale des discours sur laquelle sont basés nombre de travaux des philosophes et des théoriciens du langage qui, avec des méthodes considérablement diversifiées, ont examiné au plus près le discours, c'est-à-dire la parole en acte, les mystères de son efficience, et ce, soit pour mieux le déchiffrer, soit pour l'enseigner. Quelle que soit la raison qui motive de telles analyses, un même constat les fonde: la parole ne sert pas seulement à symboliser et à raconter le monde, elle permet d'agir sur lui, et surtout d'agir sur autrui, pour le pouvoir comme pour le partage du sens. Comme tout discours, la rumeur est un récit dont la visée argumentative, manifeste ou latente, réalise le partage du sens de l'événement faisant l'objet de rumeur.

Parole en acte, la rumeur viserait davantage l'action sur le destinataire que les moyens rhétoriques mis en place pour la réussite de l'argumentation bien que l'effet dépende des moyens -, comme le ferait, par exemple, la plaidoirie juridique. Quelle est alors la place de la rumeur dans la typologie des formes de discours? Avant de répondre à cette question, faisons un détour par le rappel des formes de discours importantes fixées par l'usage dans le discours social, terme que Marc Angenot (1989) désigne comme tout ce qui se dit et s'écrit dans un état de société, c'est-à-dire tout ce qui s'imprime, tout ce qui se parle publiquement ou se présente aujourd'hui dans les médias ou sur Internet. Enfin, tout ce qui se narre et s'argumente, si l'on pose que la narration, la description et l'argumentation sont les trois grands modes de mise en discours, car chaque discours correspond à des pratiques et à des énoncés clairement distincts. Du point de vue sémiotique, pour qu'il y ait un récit narratif, il faut un sujet du faire, dont l'action transforme les états de la situation initiale à la situation finale (Courtés, 1976). En revanche, la description prend normalement appui sur des énoncés d'état et de perception (être, paraitre), équipés de qualifications (Fontanille et Greimas, 1991).

Parmi ces formes de discours, l'argumentation a un statut à part. D'une part, elle répond elle aussi à des types d'énoncés qui lui sont propres, ne serait-ce que par des connecteurs logiques du type «par conséquent", "par ailleurs", "en revanche» ou "puisque", qui pourraient à eux seuls constituer les formes du discours argumentatif, même si le contenu n'y était pas. Toutefois, les mécanismes de l'argumentation se rencontrent volontiers à travers les autres types de discours, car il est possible de raconter pour justifier ou décrire pour dénoncer. Certes, divers domaines du discours sont particulièrement impliqués par l'argumentation - le plaidoyer juridique, le discours politique -, mais si on envisage très largement la dimension persuasive, et si on la considère comme inhérente à tout échange de parole, fût-il simplement informatif, on peut admettre que l'argumentation est transversale aux différents types de discours et qu'elle les enrobe. Toute activité de langage peut dès lors être considérée comme argumentative dans la relation de confiance, de défiance, de persuasion, de séduction, ou même d'émotion qu'elle instaure entre les partenaires de l'énonciation: l'énonciateur et le destinataire. On parle souvent d'un principe de coopération qui est à la base de toute entrée dans un dialogue (Eco, 1985). Denis Bertrand considère alors que "tout échange verbal, immédiat à l'oral ou différé à l'écrit, suppose entre les interlocuteurs un contrat énonciatif qu'on appelle aussi, plus précisément, un «contrat fiduciaire" (1999: 18). Car «ce mot, issu du latin fiducia, la confiance, indique bien le noyau de tout échange» (ibid.).

Chaïm Perelman (1977) montre que l'argumentation consiste à étudier les techniques de discours qui permettent de provoquer ou d'accroître l'adhésion d'un auditoire aux thèses qu'on présente à son assentiment. Entre-temps, la linguistique avait occupé son territoire en développant la pragmatique, théorie de la parole comme acte qui, par là même, a intégré l'argumentation (Austin, 1970). Au cour de la 
rumeur, il y a une dimension argumentative basée sur le dialogue entre les deux pôles de la communication que sont l'énonciateur et le destinataire. Car la notion de rumeur est équivoque: d'un côté, elle laisse supposer qu'une information véhiculée n'a qu'une valeur secondaire, mensongère, douteuse, alors qu'en pratique, puisque cette information circule dans la sphère publique sous forme d'une nouvelle digne d'être racontée, elle procède du contrat fiduciaire entre les interlocuteurs dont l'énonciation sur la nature de la nouvelle rumorale vient elle-même de l'énonciation collective (Bertrand, 1993). En effet, la persuasion est le processus par lequel on convainc quelqu'un de croire ou de faire quelque chose. Quand on entend une rumeur, on ne l'interprète que sous un certain angle. Selon le contexte, la signification de la rumeur change. Sa compréhension paraît ainsi imparfaite, toujours soumise à l'orientation d'un point de vue et du contexte d'énonciation qui prend en charge la nouvelle jugée comme rumeur. On supplée toujours les parties manquantes par l'habitude de voir et par la confirmation de la parole d'autrui, parole axée sur les valeurs en circulation dans la société. La rumeur, parole individuelle colportée de bouche à oreille, est susceptible de cristalliser l'opinion publique. Sa charge affective, caractérisée plus par son caractère inattendu que par son contenu dramatique, en facilite la propagation et les diverses transformations qu' elle subit au cours de sa traversée du discours social.

Ainsi, pour être racontable, une rumeur doit comporter en elle-même une certaine dose de singularité et de curiosité qui la rendra appréciable et reconnaissable comme phénomène insolite par un destinataire sur qui le message rumoral doit agir. Elle doit argumenter sur les valeurs dans la société, les rapports humains qui sous-tendent celles-ci, établissant ainsi un contrat fiduciaire entre le destinateur et le destinataire. Elle suppose non seulement la communication d'une nouvelle insolite, mais aussi un cadre narratif et discursif spécifique dont le destinataire reconnaîtra aisément. Sans cette forme spécifique, cohérente et racontable, les actes ou les situations évoqués s'évanouiraient dans le flux interrompu d'autres actions, visions et situations qui marquent la quotidienneté du genre humain à travers le discours social. La rumeur est donc avant tout une construction à la suite d'une opération intelligente qui est scandée par des phases de repérage, de sélection, de présentation, de contextualisation et de narration du contenu brut de la nouvelle. Elle dépend d'une situation d'énonciation dans laquelle son contenu se négocie entre le narrateur et le récepteur. Une telle situation est par ailleurs surdéterminée par les préjugés et les clichés dont se nourrit aisément la rumeur. Ceux-ci sont au cœur de la vie sociale comme schéma dans lequel se trouvent thésaurisées différentes figures figées et constamment ressassées par de multiples énonciations individuelles. Ce sont des expressions d'idées reçues, admises par les individus en fonction de leur adhésion implicite aux jugements circulant dans leur groupe de référence en dehors de toute expérience personnelle; adhésion qui crée, sur le plan de la communication, un seuil d'acceptabilité entre les membres de ce groupe. La circulation de la rumeur se fait grâce à l'existence de ce seuil d'acceptabilité des préjugés et des stéréotypes en cours dans une société et qui préexistent aux énonciations individuelles qui les convoquent.

Ces formes figées nourrissent la rumeur, puisque, souvent de nature passionnelle, ces figures sont d'ordre psychologique et échappent ainsi au jugement critique et moral des interlocuteurs. Un tel seuil d'acceptabilité de la solidarité sociale intragroupale qui nourrit la rumeur peut révéler, dans certains cas, la cristallisation de la haine et le mépris pour le groupe adverse. Construit par le récit, le contexte d'énonciation détermine la portée et l'amplitude de la rumeur, sa valeur et son intensité médiatique. Cela d'autant plus que ce n'est pas la réalité des faits colportés qui compte, mais bien davantage le prestige social de l'informateur, le canal utilisé et la charge affective de l'information. En effet, plus celle-ci est importante, aussi bien par son caractère inattendu que par son contenu dramatique, plus elle a de chance de prendre corps dans le discours social. Car, puisqu'elle 
est un mode de communication privilégié d'expression sociale, la rumeur se propage en nommant les coupables pour en faire le bouc émissaire de l'angoisse collective. Elle acquiert alors une fonction délatrice des ennemis, que les usagers se construisent grâce à des polarisations sociales en cours dans une société.

En convoquant les clichés et les stéréotypes déposés dans la mémoire collective, la narration rumorale vise l'adhésion des interlocuteurs de l'espace public sur la réalité de la menace évoquée et, par conséquent, la focalisation de l'opinion sur un seul élément. Ce faisant, l'énonciation rumorale crée un contexte axiologique binaire, marquant de manière simple et claire la frontière entre le bien et le mal, l'acceptable et le condamnable. Ce monde dichotomisé entre les bons et les méchants réalise le contexte d'énonciation de la rumeur sur le «complot des Tutsi " ${ }^{2}$, qui constituera le corpus d'illustration au cadre théorique évoqué plus haut. Au départ, une guerre civile. Les maisons sont brûlées, le bétail et les champs détruits. L'exil des Tutsi commence. Par vagues. 1959. 1960. 1963. 1964. Dans le discours social du Rwanda, une rumeur naît. Les Tutsi chassés du pays préparent leur retour armé. Le «complot tutsi» est né: il aura une vie longue. La rumeur est alimentée par le discours politique. Une crise. C'est la cause du Tutsi. En 1990, la rumeur devient réalité. Organisé en mouvement politique, le Front patriotique rwandais (FPR), la communauté exilée vivant dans les pays limitrophes, veut rentrer de force au pays. La guerre durera quatre ans. Le 6 avril 1994, le général Habyarimana, président du Rwanda à l'époque, est tué dans un accident d'avion. Jusqu'ici aucune enquête n'a jamais été faite, ni par la France, qui a perdu les pilotes et l'équipage de l'avion présidentiel, ni par le gouvernement actuel du Rwanda. Les Tutsi de l'intérieur du pays subiront le génocide le plus rapide de l'histoire des génocides. En deux semaines, 90\% des Tutsi sont tués. Le génocide prend fin avec la victoire du FPR sur les Forces armées rwandaises (FAR), qui avaient réalisé le génocide en juillet 1994. Voilà le fond de cette macabre rumeur. Je voudrais analyser le sens de cette rumeur dans le contexte du Rwanda durant la période du génocide d'abord et dans celui du Rwanda postgénocide ensuite. En comparant les deux usages d'un même continuum rumoral, je voudrais établir les différences de signification suivant les deux contextes.

\section{LE «COMPLOT TUTSI» DURANT LE GÉNOCIDE OU LA CROYANCE COMME VÉRITÉ}

Au début des années 1990, la rumeur sur le «complot tutsi» reprend de façon plus virulente à la suite de la guerre de 1990. Elle est relayée par les médias d'État et les journaux privés, dont Kangura, qui allait publier Les Dix Commandements d'un $\mathrm{Hutu} \mathbf{u}^{3}$ :

1. Tout Muhutu doit savoir que Mututsikazi ${ }^{4}$, où qu'elle soit, travaille à la solde de son ethnie tutsi. Par conséquent, est traitre tout Muhutu qui épouse une Mututsikazi, qui fait d'une Mututsikazi sa concubine, qui fait d'une Mututsikazi sa secrétaire ou sa protégée.

2. Tout Muhutu doit savoir que nos filles Bahutukazi sont plus dignes et plus conscientes dans leur rôle de femme, d'épouse et de mère de famille. Ne sont-elles pas jolies, bonnes secrétaires et plus honnêtes!

3. Bahutukazi, soyez vigilantes et ramenez vos maris, vos frères et vos fils à la raison.

4. Tout Muhutu doit savoir que tout Mututsi est malhonnête dans les affaires. Il ne vise que la suprématie de son ethnie.

Par conséquent, est traitre tout Muhutu

- qui fait alliance avec les Batutsi dans ses affaires;

- qui investit son argent ou l'argent de l'État dans une entreprise d'un Mututsi;

- qui accorde aux Batutsi des faveurs dans les affaires (l'octroi des licences d'importation, des prêts bancaires, des parcelles de construction, des marchés publics).

5. Les postes stratégiques tant politiques, administratifs, économiques, militaires et de sécurité doivent être confiés aux Bahutu.

6. Le secteur de l'enseignement (élèves, étudiants, enseignants) doit être majoritairement hutu.

7. Les Forces armées rwandaises doivent être exclusivement hutu. L'expérience de la guerre d'octobre 1990 nous l'enseigne. Aucun militaire ne doit épouser une Mututsikazi.

8. Les Bahutu doivent cesser d'avoir pitié des Batutsi. 
9. Les Bahutu, où qu'ils soient, doivent être unis, solidaires et préoccupés de leurs frères Bahutu.

- Les Bahutu de l'intérieur et de l'extérieur du Rwanda doivent rechercher constamment des amis et des alliés pour la Cause hutu, à commencer par leurs frères bantous.

- Ils doivent contrecarrer la propagande tutsi. Les Bahutu doivent être fermes et vigilants contre leur ennemi commun tutsi. 10. La révolution sociale de 1959, le Référendum de 1961 et l'idéologie hutu doivent être enseignés à tout Muhutu et à tous les niveaux. Tout Muhutu doit diffuser largement la présente idéologie. Est traître tout Muhutu qui persécutera son frère Muhutu pour avoir lu, diffusé et enseigné cette idéologie.

Le texte s'ordonne autour de deux thèmes: la méchanceté du Tutsi et la naïveté du Hutu. Les deux ennemis sont campés face à face avec des alliés et des ennemis respectifs. Le message général de l'argumentation est d'amener le Hutu à hair le Tutsi. Sur le plan formel, le texte est divisé en deux types de lois: celles qui donnent le savoir à acquérir au Hutu (parcours cognitif) et celles qui définissent l'action tirée du savoir (parcours pragmatique). Le texte adopte la structure d'une mélopée traditionnelle des travailleurs en groupe, construite selon un rythme binaire d'appel et de réponse:

Appel: «Tout Muhutu doit savoir que...»

Réponse: «Par conséquent...»

Une telle structure se trouve, la plupart du temps, dans une même loi ( $\left.n^{\circ} 1, n^{\circ} 2 n^{\circ} 4, n^{\circ} 10\right)$. Les quatre premières lois donnent un savoir et précisent l'action à mener: le Tutsi est méchant, il faut l'exclure. Les autres lois sont des réponses aux appels des lois précédentes. Ainsi, la loi no3, qui s'adresse aux femmes hutu, constitue une action pragmatique à l'appel lancé dans la première partie de la loi no 2 . Selon cette loi, les qualités morales d'une femme hutu en font une épouse modèle par rapport à la femme tutsi, déjà qualifiée négativement dans la loi $\mathrm{n}^{\circ} 1$.

La partie «appel» du décalogue est marquée surtout par les verbes de la cognition, essentiellement le verbe savoir suivi de la modalité du devoir-faire, qui constitue justement un leitmotiv qui scande l'action:
"Tout Muhutu doit savoir...». Aussi le texte évoque-t-il constamment le «complot tutsi» qui vise à ravir le pouvoir politique au groupe hutu, notamment par l'entremise de la femme tutsi, dont l'image est celle de la femme fatale évoquée dans le discours populaire sous le terme ikizungerezi (littéralement «femme qui fait tourner la tête puisque désirable car belle»); d'où l'injonction du journal aux Hutu: «nos filles Bahutukazi [...] Ne sont-elles pas jolies?». Cette évocation fait référence à une série de rumeurs sur la traitrise de femmes tutsi travaillant à la fonction publique grâce au soutien de leur mari ou de leur amant et qui livrent les secrets d'État à l'ennemi. Elle est également en relation avec une autre pratique administrative qui empêchait les militaires, du moins les officiers rwandais de la première et de la deuxième République (tous Hutu, à une exception près) d'épouser une femme tutsi. La rumeur sur ce complot tutsi s'inscrit ainsi dans une argumentation dont la visée est un appel à l'ordre, au respect de l'interdit: «tout Muhutu qui fait d'une Mututsikazi sa concubine, sa secrétaire ou sa protégée» viole l'interdit. Il devient «traître» (loi no 1$)^{5}$.

La rumeur selon laquelle les femmes tutsi séduisent les Hutu et les Occidentaux au service du "complot international tutsi» gagne aussi les milieux européens, ecclésiastiques surtout:

En Europe, on se passionne pour la défense des minorités et l'on passe l'éponge sur l'extermination de la majorité [...]. Les Tutsi ont réussi à inféoder, noyauter toutes les organisations internationales. Même la presse et la radio Vatican [...] où ils ont su placer des abbés rwandais tutsi [...] qui faussent toutes les informations avec une habileté extraordinaire, orfèvres de la supercherie, fourbes et maîtres es intrigues? De jolies filles tutsies rwandaises ont infiltré les organisations humanitaires et conquis le terrain par leurs charmes inégalables. ${ }^{6}$

Selon cette propagande, les Tutsi domineraient le monde grâce aux "charmes inégalables" de leurs filles, qui leur permettraient de se constituer en «un lobby international». Le mode impératif utilisé dans le texte, comme un mot d'ordre, se construit sur un énoncépivot: «les Hutu doivent cesser d'avoir pitié des Tutsi». 
La mise à mort du Tutsi se présente ainsi comme une nécessité pour la défense de la République hutu. Et pour réaliser ce projet, il faut que les Hutu se coalisent dans un parti qui défend la République, car - c'est sous-entendu - les Tutsi sont des ennemis de la République, des «féodaux». La loi $n^{\circ} 10$ tombe alors comme une conclusion logique du double parcours narratif du Hutu (savoir et action): «diffuser l'idéologie hutu» et "contrecarrer la propagande des Tutsi", sinon les tuer. Une telle structure fermée obéit à la logique de tout discours injonctif: énoncer les faits et adopter une action.

Dans ses livraisons ultérieures, Kangura convoquera le discours de la théorie des migrations, telle qu'elle a été appliquée au Rwanda par les anthropologues. Selon cette théorie, les Hutu sont des autochtones et les Tutsi des envahisseurs venus de l'étranger; les Hutu doivent s'unir pour combattre leur ennemi commun ${ }^{7}$ :

Les Tutsi nous ont trouvés au Rwanda, ils nous ont opprimés et nous l'avons supporté. Mais maintenant que nous nous étions sortis du servage et qu'ils veulent réinstaurer la chicote matinale, je pense qu'aucun Hutu ne pourra le supporter. La guerre que mène Gahutu est juste, c'est un combat pour la république. Que tous les Hutu le sachent, lorsque les féodaux arriveront au Rwanda, ils ne feront pas de distinction entre Hutu du Nord et ceux du Sud, qu'ils sachent que ce sera la fin à eux tous.

Ailleurs, il est affirmé que les Tutsi occupent les villes des pays des Grands Lacs, de l'Ouganda au Congo, en passant par le Rwanda et le Burundi ${ }^{8}$ : Savais-tu que la ville de Kigali est habitée par les Tutsi à 85\%? Lorsqu'on a renvoyé de la ville tous ceux qui n'y avaient pas de travail, seuls les Hutu sont partis. Quant aux Tutsi, ils ont pu obtenir des attestations de travail grâce à leurs frères qui affirmaient qu'ils les utilisaient comme domestiques. Ajoutez qu'après leur libération, les complices sont venus s'entasser à Kigali afin de s'assurer une meilleure protection de la communauté internationale. Qu'est-ce qui manque pour que les Hutu soient aussi solidaires? Si les Hutu ne font pas attention, il seront bientôt renvoyés à la campagne en laissant les Tutsi peupler seuls, les villes. Regardez Kigali, Bujumbura, Kinshasa et Kampala...
Dans son ressentiment à l'égard des Tutsi, le journaliste réalise ainsi un enthymème, dont le développement implique, comme prémisse majeure, une théorie générale du complot ("complices») des Tutsi pour dominer les Hutu. Ici, l'argumentation assure la circulation de la rumeur selon laquelle les Tutsi vont reprendre le pouvoir politique et renvoyer les Hutu à la campagne. Cela suppose un savoir partagé sur la nocivité du Tutsi au sein de la communauté hutu, lectrice du journal Kangura.

La rumeur propagandiste de complot contre les Hutu s'internationalise. Dans la guerre du Rwanda, la communauté internationale aurait pris parti pour les Tutsi du FPR 9 . Ici, la rumeur s'appuie sur le stéréotype selon lequel les Tutsi sont intelligents et habiles politiquement pour diviser les Hutu et rallier la communauté internationale à leur cause.

Les ordinateurs du monde entier avaient laissé croire au FPR et aux Tutsi qu'ils allaient la gagner et conquérir le pays en quelques jours. D'ailleurs, quand tu considères les armes de grande capacité qu'ils avaient, quand tu songes qu'ils avaient réussi à mettre la Communauté internationale de leur côté et qu'ils étaient parvenus à transformer l'intérieur du pays en repaire de déchirement entre les $\mathrm{Hutu}$, tu comprends qu'ils s'étaient préparés d'une manière qui dépasse tout ce que l'on peut imaginer; et que rien ne peut les empêcher d'arriver à leur fin.

Ce discours de l'encerclement par l'ennemi tutsi devient de plus en plus l'argument massue donné par les idéologues hutu pour rallier tous les Hutu à leurs idées. Et les traitres hutu sont pointés du doigt en vertu du décalogue. Il s'agit de conduire tous les Hutu à agir comme une masse, dont l'horreur est la règle. Cette idéologie de la "majorité» corporelle, proche de l'idée de foule, a conduit beaucoup de Hutu et leurs alliés pères blancs, reconnus pourtant pour leur honorabilité, à hurler à la mort du "cafard", à saccager, à dénoncer et à tuer!

Par ailleurs, les articles de Kangura adoptent une propagande de la contrevérité qui frise souvent le contresens, comme dans le discours du Parmehutu au début des années 196010 - quand on sait effectivement que les Tutsi sont marginalisés par une 
politique de ségrégation qui leur octroie le maigre $10 \%$ des places disponibles dans les écoles secondaires et supérieures ainsi que dans la fonction publique et le secteur privé, alors qu'ils représentent $25 \%$ de la population totale.

Même si conformément à leur nature, ils nous ont fait $d u$ mal en guise de reconnaissance, les Tutsi n'étaient exclus d'aucun poste dans le gouvernement du pays. Sous la première République, les Tutsi n'ont pas participé au pouvoir. Ils auraient soumis notre jeune République aux corvées de la houe. Cela ne veut pas dire qu'il était exclu qu'ils puissent collaborer avec les autres Rwandais. Son excellence Grégoire Kayibanda leur a toujours demandé de diminuer leur orgueil et de s'entendre avec les autres Rwandais. Quand vint la deuxième République, celle de l'unité qui mit fin au gouvernement de Kayibanda, les Tutsi furent favorisés de façon manifeste. Si leur manque d'intelligence ne les avait pas poussés à montrer leur cruauté en épuisant Habyarimana et en tuant ses enfants. Il est en effet le père de tous les Rwandais qu'il a rassemblés dans le mouvement MRND. Ils dominent l'éducation nationale, la Justice est leur domaine réservé, dans l'administration [...].

Dans les allées d'un pouvoir qui se voulait hutu à Kigali, l'objectif était déjà, à la parution du premier numéro de Kangura en 1991, d'en finir avec le groupe tutsi. D'autres médias vont diffuser systématiquement cette rumeur sur le complot tutsi. L'Office rwandais de l'information (ORINFOR) veille à ce que la presse écrite et Radio Rwanda relaient les appels aux meurtres à peine déguisés des discoureurs publics, politiques ou militaires qui évoquent à longueur de journée la rumeur du complot des Tutsi. Dans les couloirs de la maison présidentielle, certains idéologues concoctent, en février 1992, un nouveau parti, la Coalition pour la défense de la République (CDR), chargée de promouvoir au grand jour l'expression la plus haineuse d'une pensée unique radicale - celle du pouvoir - par la création de la RTLM (Radio-Télévision des Mille Collines) en 1993.

Après l'assassinat de Juvénal Habyarimana le 6 avril 1994, c'est en effet ouvertement d'extermination qu'il s'agit. Cette radio a joué un rôle déterminant dans des collines étroitement quadrillées et encadrées, mais aussi psychologiquement préparées à écouter la radio comme parole d'Évangile et à en déchiffrer les messages, qui valent permis puis ordre de tuer. C'est en suivant ce schéma initial de transformation d'une rumeur - le complot des Tutsi - en information que l'attentat contre le général Habyarimana sera attribué aux Tutsi et présenté comme l'élément déclencheur du génocide des Tutsi. En soi, il est évident que l'épisode décrit - l'assassinat d'un chef d'État, fût-il dictateur - comporte une singularité qui peut capter l'attention de lecteurs avides de sensationnalisme et habitués à en consommer sur le thème de la violence en Afrique ou sur le cas spécifique du génocide du Rwanda. Ce qui est particulier, ce qui en fait une rumeur, c'est d'attribuer la responsabilité aux Tutsi et de les désigner, par conséquent, comme responsables du génocide qui les a visés et qui a emporté le quart de la population tutsi du Rwanda entre avril et juillet 1994. La rumeur fait de la victime le bourreau de luimême. La rumeur se construit à partir de représentations sociales comme les préjugés, qui cristallisent l'angoisse collective sur la figure du bouc émissaire qui est ici le Tutsi.

Dans les médias rwandais de cette période, avant et pendant le génocide, le recours à la rumeur du "complot tutsi» semble obéir à la technique de la propagande très connue de l'appel à la peur. Le journaliste ou le politique recherchent l'adhésion du groupe hutu en inspirant la peur dans l'opinion publique pour justifier ses forfaits vis-à-vis des citoyens tutsi. La fonction de la rumeur est ici la délation. Tout citoyen hutu se comporte en délateur vis-à-vis des pouvoirs publics entre les mains de l'élite hutu et transforme le citoyen tutsi en traître. La rumeur se limite ici au cadre national, où elle participe au contexte idéologique opposant les partis politiques entre les nationalistes et les ethnistes comme le Hutu Power. La rumeur partage le monde du bien et le monde du mal. Son destinataire est donc le Rwandais plutôt hutu nationaliste, qu'on voudrait voir changer de position, ou le Rwandais ethniste, dont on voudrait radicaliser les positions et les actions. La rumeur délatrice a pour fonction de sensibiliser 
l'opinion publique, déjà imprégnée de préjugés, et de polariser l'attention sur le but que le narrateur s'est fixé. Une fois atteint le niveau émotionnel souhaité, on déclenche une nouvelle et l'on désigne le bouc émissaire. En étudiant en profondeur les différentes formes de cette rumeur, on remarque que les acteurs rwandais n'établissent pas une distinction entre la manipulation politique par les médias d'État et leur croyance en ce complot tutsi. Ils croient en celui-ci et admettent en même temps le rôle de la manipulation politique par les autorités. Comme s'ils ne croyaient pas aux mythes collectifs tout en y adhérant: la rumeur ne relève pas de la fiction, comme on pourrait le croire, elle relève des récits considérés comme vrais, des légendes cosmogoniques aux mythes de fondation, en passant par les paroles des politiciens!

\section{LE «COMPLOT TUTSI» APRĖS LE GÉNOCIDE OU L'ART DE LA VARIATION SUR LE VRAI}

Nous pourrons voir, dans le recyclage de la rumeur $\mathrm{du}$ "complot tutsi» après le génocide, une stratégie argumentative visant à faire partager la «barbarie» entre les Hutu et les Tutsi. Le destinataire est évidemment l'Occidental et le Rwandais hutuisant qui souhaitent la mainmise de la communauté internationale sur le gouvernement actuel du Rwanda, qu'ils qualifient de «tutsi FPR». Apparaissant à des moments de grande réceptivité du public - comme lors de l'anniversaire de la commémoration du génocide des Tutsi -, la rumeur semble caractérisée par la mobilisation émotionnelle générale, qui aboutit à une action de masse, en l'occurrence le génocide. Celui-ci repose donc sur l'exacerbation de la différence ethnique, religieuse, raciale ou politique dont se nourrit la rumeur en temps de guerre. Le meurtre dépasse le cadre fortement symbolique de lynchage, il est l'expression ultime et radicale du besoin collectif de détruire.

Il a été montré que la rumeur, selon laquelle les Tutsi avaient causé le génocide d'eux-mêmes, avait le plus souvent maquillé certains faits et en avait délibérément omis d'autres. On estime en conséquence que cette rumeur est le fait de la propagande d'extrémistes qui, voulant lier le génocide, commence par dire que si les Tutsi sont responsables de la mort des leurs, il n'y a pas eu de planification par le gouvernement du général Habyarimana. La négation du génocide vise de facto l'obtention d'un non-lieu pour ce qui est admis comme un crime. En l'absence de l'organisation préméditée du crime, il n'y a plus ni criminels ni victimes du génocide. On parle dès lors de la légitime défense, par le massacre spontané des Tutsi, de leurs concitoyens et voisins Hutu. Cette rumeur-négation du génocide, répandue en dehors du Rwanda, et particulièrement en France, vise surtout un objectif politique. Au gouvernement français, elle permet de préserver longtemps son influence en maintenant l'image qui fait de la France l'amie de l'Afrique, image ternie justement par la collaboration de ce pays ave un régime ouvertement raciste, qui utilise notamment des cartes d'identité ethnique, comme l'étoile jaune à l'époque des nazis. À la diaspora politique rwandaise d'Occident, la rumeur permet de nier le génocide des Tutsi pour justifier les actions militaires contre le régime de Kigali, qualifié de régime tutsi, et, par conséquent, de réactiver le thème du complot tutsi envahisseur et responsable du mal de l'Afrique de la région des Grands Lacs d'Afrique orientale. Outre les manipulations ordinaires, la rumeur du complot tutsi nie, par son évocation même, le fait historique qu'est le génocide des Tutsi par le Hutu Power. Certains diront, comme le fait le colonel Théoniste Bagosora ${ }^{11}$, porte-parole de ce mouvement au cours des années qui ont suivi le génocide, et orchestrateur durant trois mois des massacres des Tutsi au pays, que le génocide des Tutsi est une construction des Tutsi eux-mêmes. Et les différents témoins au procès des présumés coupables du génocide des Tutsi sont présentés comme autant d'agents manipulateurs du Département de renseignements intérieur de Kigali (DMI), et leur faible nombre (et pour cause) comme une preuve du caractère secondaire de l'événement. Cette rumeur se situe donc dans une logique beaucoup plus vaste de la théorie du complot, qui interprète des événements comme le résultat de 
manipulations par une ou plusieurs puissances secrètes ou d'une conspiration, dont les fonctions argumentatives ont été déjà analysées dans les médias nationaux.

Reprise constamment par les médias, la rumeur acquiert une nouvelle signification; elle est souvent adressée aux gens qui sont déjà bien disposés à l'assimiler, comme l'ont noté avec justesse Jean-Paul Gouteux (1999, 2002), Isabelle Gaudin (1996) ou Patrick de Saint-Exupéry (2004), en montrant comment certains politiques et journalistes ont utilisé en France cette rumeur du complot tutsi pour rendre les Tutsi responsables du génocide qui les a frappés. Ces auteurs et bien d'autres ont analysé les mécanismes de «désinformation et manipulation sur le génocide rwandais». Ils ont fait remarquer, par exemple, que le célèbre Le Monde est allé jusqu'à publier de fausses informations dans sa livraison du 10 mars 2004 en affirmant, d'une part, que c'est le président Paul Kagame du Rwanda - qui, à l'époque du génocide, était le chef militaire de la guérilla du FPR - qui a commandité l'assassinat de son prédécesseur et, d'autre part, que la boîte noire du Falcon 50 de Habyarimana avait été oubliée pendant près de 10 ans au siège de l'ONU, à New York. Or, d'après les résultats de l'enquête menée par le secrétaire général de l'ONU et publiés le 7 juin sur le site Internet de l'Organisation, le Cockpit Voice Recorder (CVR), conservé sous clé au Département des opérations de maintien de la paix (DOMP), n'est pas celui du jet présidentiel, et ne peut donc pas être considéré comme une pièce à conviction dans l'enquête sur l'attentat du 6 avril $1994^{12}$. Malgré cela, la rumeur court! Car l'intentionnalité de la rumeur est de faire paraître vrai le complot des Tutsi. Tous les moyens sont bons, alors.

En incriminant le FPR comme étant le mouvement des Tutsi responsable de l'attentant contre l'avion du général Habyarimana, on en fait le responsable du génocide; en suivant le même raisonnement, on fait du génocide des Tutsi une manipulation tutsi et l'on absout indirectement les responsables du génocide. C'est le sens de la rumeur, qui va d'ailleurs dans la ligne argumentative des présumés coupables du génocide en prison et en attente de leur procès, lesquels voudraient que le génocide des Tutsi dont ils sont accusés soit présenté à l'opinion publique internationale comme le résultat de la fureur populaire déclenchée par l'assassinat du général Habyarimana. Par ailleurs, la reprise de la rumeur dans les médias, dans les discours politiques ou dans les livres, a une autre visée argumentative: elle consiste à dire que les Tutsi du FPR ont également commis le génocide des Hutu. Toutes les imperfections du Rwanda actuel, tous les délits et les crimes qui s'y commettent sont attribués au FPR, dont on fait le pendant du Hutu Power. On parle alors de double génocide, de génocides ou d'extrémismes inverses et symétriques. Interrogé par les journalistes sur le pluriel du mot génocide dans la version écrite de son discours, le président français - François Mittérand - répondait déjà en 1994: «Voulez-vous dire que le génocide s'est arrêté avec la victoire des Tutsi? Je m'interroge aussi» ${ }^{13}$, même s'il n'existait aucune trace d'un second génocide après une enquête par les Organisations des droits de l'homme après cette rumeur. Peu importe, les attaquants du FPR laissent planer le soupçon en évoquant l'intelligence supérieure et la fourberie des Tutsi qui leur auraient permis de réaliser le crime sans laisser de trace. Banalement, la rumeur devient ici un argument dans une visée propagandiste, car une telle rhétorique de la diabolisation du FPR et des Tutsi se situe dans la logique des complices internationaux du génocide des Tutsi. Ils deviennent ainsi d'honnêtes médiateurs entre deux «barbaries», l'une hutu et l'autre tutsi. Cela concerne directement la France, pour sa politique d'aide au régime du général Habyarimana qui a organisé le génocide des Tutsi, mais aussi l'ONU, coupable d'acceptation passive et de nonassistance aux victimes du génocide qui se commettait en direct devant les télévisions du monde entier. Cette rumeur de la responsabilité du tutsi victime devenu bourreau n'a aucune limite, même pas celle de la vraisemblance. Tout se passe comme si le génocide des Tutsi commis par des Hutu étant avéré, il faudrait 
rétablir l'équilibre en chargeant les Tutsi du FPR de crimes équivalents. On parle alors de massacres entre les Rwandais ou de guerre interethnique. La rumeur vise ici à obtenir la désapprobation de la part du destinataire sur son évaluation du gouvernement de Kigali après le génocide. En présentant les Tutsi victimes du génocide sous les traits de leurs bourreaux - les Hutu Power -, la rumeur suggère le mépris vis-àvis de deux groupes chez le lecteur-cible, en puisant ainsi dans le vaste vivier des stéréotypes occidentaux sur l'Afrique, notamment ceux du courant afropessimiste. Ici, les médias et les essais expliquent que l'Afrique non seulement était tombée au plus bas, mais surtout qu'elle ne pourrait plus s'en remettre, qu'on était en train d'assister à l'agonie d'un continent en quelque sorte prêt à être rayé de la carte géopolitique internationale. Ce que Stephen Smith affirme sans ambages:

Le présent n'a pas d'avenir en Afrique. Tel était notre point de départ. À l'arrivée, la démonstration a été faite. Elle est écrasante, déprimante, irrécusable. Le continent se meurt.

(2003: 227)

Smith établit le diagnostic de la nécrologie africaine:

Pourquoi l'Afrique meurt... C'est désormais la seule question qui reste, l'unique qui importe, vitale pour les Africains, fondamentale pour les autres, du moins ceux qui cherchent toujours à comprendre ce continent, "Ubuland» sans frontières, terre de massacres et de famines, mouroir de tous les espoirs.

Il va sans dire que dans une telle vision afropessimiste, toute organisation, tout parti politique, ne fait que préparer ou parachever le "suicide collectif" de l'Afrique (ibid.). Aucune exception. Le FPR et ses dirigeants ne sont autre chose que des «kmers noirs». Des bourreaux en tout point semblables au Hutu Power; peu importe s'ils ont arrêté le génocide ${ }^{14}$. Ils ne peuvent pas prétendre au statut de "sauveurs» de rescapés du génocide: ils sont africains, donc barbares comme leurs congénères du continent! La réussite de ce récit du complot tutsi dépend donc du contexte de réception que les journalistes, hommes politiques et écrivains convoquent pour s'adapter aux lieux communs en cours dans la société occidentale sur l'Afrique. La rumeur s'adapte ainsi au lectorat en usant d'une rhétorique basée sur les lieux communs disponibles dans le vivier collectif des stéréotypes, forme d'énoncés figés et déposés dans le thésaurus du discours social.

Par ailleurs, cette rumeur du complot tutsi se recycle à travers différents courants d'idées en Occident, où s'amalgament, sur fond de communautarisme, dans une soupe imaginaire fétide, la théorie du complot juif, la dénonciation de l'impérialisme américain, l'anticapitalisme primaire et divers registres du négationnisme, réaménagés aux goûts de la gauche actuelle en quête de la nouvelle solidarité internationale. En gros, la stratégie est de dénoncer le «complot tutsi» en l'amalgamant avec le "complot juif", en disant que le génocide des Tutsi a été inventé par les capitalistes américains selon «un plan d'assujettissement et de neutralisation du Congo et de l'Afrique " ${ }^{15}$. La visée argumentative sert à soutenir des idées qui sont ouvertement pronazies en usant de la rumeur du complot tutsi. Une manœuvre rhétorique de diversion, certes, mais qui n'atteint pas moins sa cible. Ce courant a des adeptes également en Afrique où certains médias, comme à Kinshasa, utilisent le complot tutsi de la même manière que le faisaient le journal Kangura ou la RTLM. Ici le génocide n'est qu'un prétexte inventé par les Américains pour avoir la mainmise sur le Congo par les Tutsi du FPR interposés, comme le note Donatien Banota:

Pourquoi ce qui marche au Moyen-Orient ne devrait-il pas s'appliquer ailleurs et notamment en Afrique où le besoin de contrôle et de neutralisation des peuples se fait sentir...? Créer un Israël et ses Juifs devenait un objectif et un préalable pour le contrôle de l'Afrique. (2000: 1)

Selon l'auteur, ce plan nécessitait un génocide que «les États-Unis prévenants s'engageaient à fournir cousu main aux Tutsi» (ibid.). Le génocide des Tutsi est un cadeau des Américains aux Tutsi! D'autres ne parlent-ils pas du génocide comme «fonds de 
commerce» du gouvernement de Kigali? «On n'est pas l'élu de Dieu gratuitement", continue Banota. Il

poursuit sa comparaison des complots juif et tutsi:

Les Hima-Tutsi présentaient quelques similitudes avec les Juifs

(le même esprit de caste fermé, la même volonté d'organisation,

de discipline et de solidarité...) [...]. Tout comme les Juifs sémites, les Hima-Tutsi sont en conflit permanent avec tous leurs voisins.

[...] De même, ils ont tendance à essaimer systématiquement

dans tous les pays dont ils revendiquent ensuite la nationalité

tout en conservant leur allégeance au Rwanda. (Ibid.)

Même si le complot américain protutsi relève plus

du fantasme ou de l'intoxication des services de

renseignements des pays hostiles au Rwanda, la

rumeur continue son chemin. Car les Américains, qui

étaient au courant du génocide préparé puis mené au

Rwanda, ont choisi l'inaction et l'alignement passif

sur la politique française, qui soutenait le régime qui a planifié puis organisé le génocide ${ }^{16}$. C'est dire que la rumeur est dotée d'une capacité à susciter en elle-

même, étant donné son caractère singulier, un intérêt chez le lecteur, et ce, en dehors de la capacité d'un rédacteur de nouvelles ou d'un éditeur de livres à créer ou à décupler cet intérêt par ses effets de présentation ou de style. Cette nouvelle, pour devenir rumeur, serait dotée d'une valeur relative d'usage, susceptible d'être exploitée et qui se traduirait éventuellement par des bénéfices supplémentaires découlant de la mainmise sur l'État rwandais. On voit que sur le plan de la stratégie argumentative, le choix de l'efficacité prend le dessus sur la recherche de la vérité. Le complot tutsi continue d'alimenter les discours des politiques, des journalistes et des africanistes en usant simplement de l'intertextualité avec un autre énoncé circulant déjà dans le discours social: «le complot juif». La boucle est bouclée. La rumeur, devenue simple énoncé circulant sur le grand marché des discours, peut alors servir des intérêts et des stratégies des courtiers prêts à banaliser et à nier l'indicible du génocide des Tutsi pour que se maintienne la prise en charge de l'Afrique par les bien-pensants de la gauche occidentale en quête de salut international des peuples.

\section{CONCLUSION}

Dans la théorie de l'argumentation, on oppose habituellement deux tendances. La première met l'accent sur l'organisation du discours, sur les formes d'agencement de propositions qui permettent de faire progresser, de consolider et de valider l'opinion qui s'y trouve soutenue. Cette approche, par exemple, insistera sur la transformation d'une thèse que l'on réfute en une thèse que l'on propose. Elle met en regard le jeu des arguments abstraits qui la fondent et des exemples concrets qui l'illustrent sous forme de récits, de témoignages ou d'expériences. Elle examinera les modes de la citation et de la reformulation du discours d'autrui, soit pour donner un appui aux arguments, soit pour les contester. Elle mettra en évidence les différentes formes de raisonnement en jeu et cherchera à circonscrire les implicites et les relations de sens dans l'espace sousentendu entre les propositions. En somme, elle se concentre sur les moyens à mettre en œuvre pour réaliser un texte argumentatif. Le parcours narratif de la vérification utilise donc les modalités aléthiques, comme dans le discours scientifique; le sujet du discours s'efface et laisse en quelque sorte parler les choses elles-mêmes. Ces modalités conduisent ainsi à statuer sur le caractère nécessaire ou impossible, contingent ou possible de l'objet énoncé, c'est-à-dire sur la façon dont le sujet du discours procède pour que son discours paraisse urai. Quant à la seconde avenue, elle s'attache à l'action visée, à l'influence que l'on cherche à exercer sur le destinataire - lecteur, spectateur, auditeur -, dont l'argumentateur-narrateur voudrait modifier les croyances et conquérir l'adhésion aux conditions de réussite ou d'échec de l'opération. Plus largement, elle prend en compte le mode de participation du destinataire du discours à l'élaboration du sens. On considère alors, au départ, le langage étant une mise en communauté de la signification, que l'intersubjectivité est la première condition de sa réussite. Car au cœur de l'argumentation, il y a la relation avec l'autre, relation de compétition négociée par l'exercice du discours dans la perspective d'un sens partagé. La circulation de 
la rumeur se réalise dans une société fortement liée aux enjeux argumentatifs du discours. Dans les modalités énonciatives de la véridiction, le sujet du discours établit son récit, et le savoir qu'il y asserte est en relation avec les autres sujets du discours, de manière souvent conflictuelle. De telles modalités font de la rumeur plus un espace du désaccord que celui du consensus sur les valeurs sociales. Car l'énonciation rumorale se construit toujours sur le sens partagé de certaines représentations sociales, comme les préjugés et les stéréotypes vis-à-vis de certains groupes sociaux. Elle ne circule pas, par exemple, dans ces groupes minoritaires qui en désapprouvent le sens. Comme dans tout récit, l'usage de la rumeur s'inscrit dans un parcours énonciatif visant la réussite du discours, c'està-dire la manière dont l'énonciateur s'adapte à son destinataire, notamment par le choix des idées et thèses communément admises et qui n'ont plus besoin d'être énoncées et débattues. Elle procède de la coénonciation qui permet l'intercompréhension entre les trois pôles de la communication que sont l'énonciateur, le destinataire et la cible, c'est-à-dire le sujet de la culture, garant des valeurs mises en circulation par le discours. C'est dire que le discours sur la rumeur apparaît comme un lieu délicat, où s'inscrivent et se lisent la vérité et la fausseté des énoncés, le mensonge et le secret des interlocuteurs en tant que modes de la vérification qui résultent de la double sphère de l'axe de communication reliant l'énonciateur et le destinataire (le contrat de véridiction tacite entre ces deux actants de la structure de la communication). En réalité, on peut comprendre aisément que les deux voies pour appréhender l'argumentation rumorale doivent être étroitement associées, dans la mesure où ce genre de récit oblige, plus que tout autre, à considérer la conception du langage et du sens, sous-jacente à toute activité argumentative, comme un processus marqué par une inévitable incomplétude. Raconter une rumeur consiste, aussi bien en production qu'en réception, à combler les espaces vacants du sens pour s'assurer, peut-être illusoirement, d'une sorte de plénitude du sens. On colle des fragments épars pour soutenir une argumentation, comme dans l'interprétation du complot tutsi par celui du juif. La fragilité de la parole rumorale est à ce prix. Sa signification dépend du crédit que le destinataire accorde au message de l'énonciateur.

Autant dire que la croyance en une rumeur est rendue possible par le fait qu'elle relève de l'ordre de l'opinion et des valeurs dont on dispose dans la vie personnelle et sociale, et qu'elle ne saurait être perçue aussi rigoureusement que les vérités scientifiques. Et pourtant, celui qui raconte une rumeur fait valoir un jugement dans la communication quotidienne. Il raisonne; mais son raisonnement est de nature particulière. Il raisonne par l'enthymème, dont le sens étymologique - du grec thumos - désigne, d'une manière générale, l'humeur, c'est-à-dire la façon dont le sujet éprouve sa relation sensible avec le monde. Ainsi l'enthymème serait à la base d'un mode de communication qui assure le partage, sinon des humeurs, du moins des images, des opinions et des valeurs qu'on a en commun dans l'esprit. C'est parce que l'enthymème fonde ses prémisses sur les apparences partagées ou partageables, sur l'acceptable et le probable, que la narration de la rumeur en use beaucoup. Comme l'enthymème, la rumeur procède souvent d'un syllogisme incomplet. Celui qui la raconte laisse ainsi au destinataire la liberté de combler les vides du sens, en imaginant les parties manquantes qui font partie du sens commun et en participant ainsi à la construction du sens à partir des éléments implicites puisés dans le thésaurus social des stéréotypes et des préjugés. C'est que l'argumentation est un jeu complexe de rôles, elle tend à faire valoir une opinion à un destinataire universel; dans le cas de la rumeur, elle tient compte des valeurs et des opinions des cercles particuliers dans lesquels elle cherche à la faire admettre et partager. La rumeur participe de la particularisation de l'argumentation en spécifiant les valeurs en jeu entre les partenaires de la communication.

La rumeur se trouve également incorporée dans le double dispositif de l'elocutio et de l'actio, deux sources de l'énonciation. Car tout énoncé, quel qu'il soit, 
peut être rapporté à un centre qui commande l'avènement de sa mise en acte. La rumeur suppose ainsi un ancrage dans une situation d'énonciation manifestée par les marques de l'énonciateur et du destinataire. Son usage révèle par ailleurs les mécanismes sémiotiques de la modalisation, c'est-àdire ce par quoi un sujet du discours modifie, rectifie ou transforme la relation avec ce qu'il énonce. C'est par la modalisation du discours qu'une même rumeur acquiert des significations différentes, comme dans l'exemple du "complot tutsi», dont le sens variait suivant qu'il était utilisé au niveau national ou qu'il servait les enjeux internationaux en rapport avec le génocide des Tutsi du Rwanda. En effet, si les parcours modaux aléthiques caractérisent le sens de la rumeur lorsqu'elle est utilisée au Rwanda - la rumeur utilisée dans les instances publiques a un caractère vrai pour le destinataire si elle est énoncée par son groupe d'appartenance et inversement - et quand elle s'énonce dans les médias internationaux, les modalités véridictoires ou épistémiques la caractérisent. Elle acquiert le statut de discours, que l'on peut discuter, dont on peut mesurer la véracité et la limite. Dans les deux cas, la rumeur a quitté la sphère de la fiction pour être une nouvelle parmi tant d'autres, que les interlocuteurs s'échangent dans le but particulier de désigner un «bouc émissaire» aux maux qui rongent et le Rwanda et l'Afrique des Grands Lacs.

Ici la rumeur devient délation. Elle désigne l'autre groupe, l'autre communauté, l'autre ethnie dont les membres sont devenus du jour au lendemain «suspects» et "agents de la puissance ennemie». Des mots de la rumeur, on passe à l'acte: la mort. Et le survivant vit dans la terreur de cette mort possible que scande inlassablement la rumeur, mais aussi dans celle de la délation possible quand il s'est exilé plus loin de son pays natal. La rumeur devient - le jugement éthique me semble inévitable - un chancre, et le recyclage de la rumeur ne serait jamais que le déguisement d'une subtile propagande négationniste et un interdit de penser. Y a-t-il une chance de déplacer la violence sur un autre terrain de jeu, celui de l'argumentation, dans ce genre de rumeur du «complot» désignant un bouc émissaire après un génocide, quand on sait que refuser d'utiliser le mot génocide, c'est prendre partie pour le bourreau? Discursivement, certes. Sinon la dimension éthique du mot génocide est telle que l'utiliser, c'est désigner le coupable et proférer un jugement sur le bourreau. En l'absence de la dimension morale du mot, le génocide devient "massacre" ou tout simplement «autodéfense», «légitime défense» ou «barbarie réciproque entre Rwandais». Ce dramatique constat incite à prendre parti pour la vie humaine. Car la neutralité dans le génocide, rappelait Élie Weisel à une autre époque, favorise le bourreau et jamais la victime. La persistance du négationnisme du génocide des Tutsi oblige à s'interroger constamment sur la nécessité d'entretenir un devoir de mémoire afin de lutter contre l'ignorance, les préjugés et le mensonge politique. D'où la nécessité de témoigner par l'analyse de la circulation des mots qui tuent, afin que les générations futures sachent que l'Innommable a eu lieu et qu'elles puissent éventuellement défendre leurs droits par l'exercice de devoirs civiques librement compris. C'est-à-dire retrouver pour eux-mêmes le sens de l'Humain en redéfinissant des notions simples: l'Autre, la liberté, la responsabilité. Ou pour le dire comme Emmanuel Lévinas (1972), pour qui vivre, c'est se sentir responsable de l'humanité de l'autre homme. Ce jugement éthique, que je porte sur l'analyse d'un type de rumeur - celle de la théorie du complot - et sur son usage dans les meurtres collectifs comme le génocide, découle de l'examen des mécanismes de l'argumentation qui sous-tendent la rumeur dans le cas du Rwanda. Si la rumeur est un acte de langage, comme je l'ai montré, sa visée argumentative est d'exclure, voire d'exterminer quelqu'un dont la proximité est vécue comme un danger. Comme les conséquences sont réelles, l'argumentation dans une telle rumeur ramène le débat sur un autre terrain que celui de sa simple analyse: celui de l'éthique dans l'usage du discours public. 


\section{N O TES}

1. Les expressions légende contemporaine ou légende urbaine, issues de l'anglais, recouvrent la même notion.

2. Sur les stéréotypes et les idéologies qui sont à la base du génocide des Tutsi du Rwanda, voir une synthèse dans mon livre Les Récits fondateurs du drame rwandais (Semujanga, 1998).

3. Kangura, nº, décembre 1990.

4. Le suffixe kazi est la marque du féminin en kinyarwanda, la langue du Rwanda. Ainsi, Mututsikazi signifie une femme ou une fille d'ethnie tutsi, de même que Muhutukazi signifie une femme ou une fille d'ethnie hutu.

5. À propos de la figure de la femme fatale tutsi, le décalogue se trouve en relations interdiscursives et intertextuelles avec d'autres discours circulant dans le discours populaire, où l'image de la femme fatale ikizungerezi - est largement diffusée au-delà du cercle des idéologues hutu. En remontant plus loin dans le temps, l'image de la femme fatale fait son apparition à partir d'un récit dont le personnage central est la princesse Bwiza, fille de Mashira, roi des Babanda dans le royaume du Nduga, avant la centralisation du pays par la dynastie nyiginya. On disait qu'elle était tellement belle qu'elle était toujours désirable à regarder et à épouser même à l'âge avancé. Elle a fait succomber des princes du Burundi et du Rwanda. Elle en a trahi d'autres. Et pourtant, son image reste positive comme sa forme moderne, l'ikizungerezi, qui désigne à la fois une femme désirable, belle, prostituée, libertine, charmante... et traîtresse. Bref, une Dalila rwandaise.

6. ANB/BIA, no 257, 1 $1^{\text {er }}$ mai 1994.

7. Kangura, no 16 , mai 1991 (6).

8. Kangura, $\mathrm{n}^{\circ} 18$, juillet 1991 (10)

9. Kangura, n 51 , octobre 1993.

10. Kangura, n²6, novembre 1991.

11. Ce bourreau de Kigali dira tantôt que les Tutsi ne sont pas morts parce qu'on en trouve encore à Kigali; plus cynique encore, il demandera à ses juges que l'on amène les Tutsi qu'il a tués pour qu'ils se présentent à la barre et témoignent contre lui devant la justice. Sur ce personnage, voir le livre de Roméo Dallaire (2003) qui le qualifie de "diable", qui en fait une description qui est digne du titre du livre. 12. Sur la désinformation médiatique en France concernant le génocide des Tutsi, de nombreux livres et articles ont été publiés. Citons notamment les écrits de Marc Lepape (1995), de Danielle Birck et Philippe Boissette (1995), de François-Xavier Verschave (1994), de Jean-Paul Gouteux (1998, 2002), de Michel Sibton (1998) ou de Patrick de Saint-Exupéry (2004).

13. Cité par Jean-Paul Gouteux dans La Nuit rwandaise (2002: 334). 14. Un des auteurs les plus perspicaces de la diffusion de ce complot tutsi est Charles Onana (2002), dont le propos est de rendre les Tutsi responsables de l'assassinat du général Habyarimana après avoir noté subtilement que cet attentat est à l'origine du génocide des Tutsi. On est en face de la diffusion d'une rumeur usant d'un sophisme. Il en est de même du roman de Gérard de Villiers, Enquête sur un génocide (2000). Ces deux textes sont de véritables manuels virtuels sur l'utilisation de la propagande diffusant un raisonnement pour le moins étrange: les Tutsi sont responsables du génocide qui les a visés. Il ne serait pas faux de penser qu'ils sont employés explicitement pour la propagande quand on voit les moyens utilisés pour en faire la promotion. Certains auteurs n'écartent pas l'idée que de tels récits sont secrètement commandés par les services secrets français (Saint-Exupéry, 2004; Gouteux, 1999). 15. À de nombreuses reprises, le journal de l'association la Nouvelle Solidarité, Executive Intelligence Rewiew, publie des articles sur ce thème [www.larouchepub.com].
16. Cette stratégie est maintenant établie grâce aux efforts d'un organisme de recherche indépendant, la National Security Archive, qui a publié sur Internet, en août 2001, 16 documents déclassifiés consultables sur le site [www.gwu.edu].

\section{RÉFÉREN CES BIBLIO G RAPHIQ U ES}

ANGENOT, M. [1989]: 1889, Montréal, Le Préambule. Austin, J. L. [1970]: Quand dire, c'est faire, Paris, Seuil.

BANOTA, D. [2000] : «Génocide tutsi de 1994 : élément central du Plan d'asservissement du Congo ", Demain le Congo (Kinshasa), juin, [http:// users.skynet.be/wirira/genocid4.htm].

BERTRAND, D. [1999]: Parler pour convaincre, Paris, Gallimard;

[1993]: «L'impersonnel de l'énonciation. Praxis énonciative: conversion, convocation, usage", Protée, vol.21, nº 1, 25-32.

COURTÉs, J. [1976]: Introduction à la sémiotique narrative et discursive, Paris, Hachette.

CHRÉTIEN, J.P. (dir.) [1995]: Les Médias du génocide, Paris, Karthala. DAllaire, R. [2003] : J'ai serré la main du diable. La faillite de l'humanité au Rwanda, Montréal, Libre Expression.

Du Berger, J. ET M. Roberge [1989]: La Rumeur, Sainte-Foy (Québec), Célat, coll. «Rapports et mémoires de recherche du Célat", $\mathrm{n}^{\circ} 14$.

ECO, U. [1985]: Lector in fabula ou la coopération interprétative du lecteur, Paris, Grasset.

FonTANILLE, J. et A.J. Greimas [1991]: Sémiotique des passions. Des états de choses aux états d'âme, Paris, Seuil.

FroIsSART, P. [2002]: La Rumeur. Histoire et fantasmes, Paris, Belin, coll. "Débats".

GAUDIN, I. [1996]: Les Crises rwandaises depuis 1959 vues à travers La

Croix, L'Humanité et Le Monde, mémoire de maîtrise, Université Paris 1, Panthéon-Sorbonne, UFR d'histoire.

GOUTEUX, J.P. [2002]: La Nuit rwandaise. L'implication française dans le dernier génocide du siècle, Paris, Esprit frappeur;

[1999]: Le Monde, un contre pouvoir? Désinformation et manipulation sur le génocide rwandais, Paris, Esprit frappeur;

[1998]: Un génocide secret d'État (La France et le Rwanda 1990-

1997), Paris, Éd. sociales.

Greimas, A. J. [1983]: Du sens II, Paris, Seuil.

LANDOWSKI, E. [1989]: La Société réfléchie, Paris, Seuil.

LEPAGE, M. [1995]: «Des journalistes au Rwanda: l'histoire immédiate d'un génocide», Les Temps modernes, $\mathrm{n}^{\circ}$ 583, 161-180.

LÉVINAS, E. (1972): Humanisme de l'autre homme, Paris, Fata Morgana. OnANA, C. [2002]: Les Secrets du génocide rwandais, Paris, Éd. Duboiris. PeRelmAN, C. [1977]: Empire rhétorique, Paris, Vrin.

QuiLĖs, P. [1998]: Enquête sur la tragédie rwandaise 1990-1994, 4 t., Paris, Rapport de la Mission d'information de l'Assemblée nationnale, $\mathrm{n}^{\circ} 1271$.

SAINT-EXUPÉRY, P. de [2004]: L'Inavouable. La France au Rwanda, Paris, Éd. des Arènes.

SEMUJANGA, J. [1998]: Les Récits fondateurs du drame rwandais. Discours social, idéologies et stéréotypes, Paris, L'Harmattan.

SiBTON, M. [1998]: Un génocide sur la conscience, Paris, Esprit frappeur. SMITH, S. [2003]: Négrologie. Pourquoi l'Afrique meurt, Paris, CalmannLevy.

VersChAVE, F.-X. [1994]: Complicité de génocide? La politique de la France au Rwanda, Paris, La Découverte.

Villiers, G. de [2000]: S.A.S. Enquête sur un génocide, Paris, Malko production. 